\title{
Surgery of Paraclinoid Aneurysm
}

\author{
Naoki Otani, Terushige Toyooka, and Kentaro Mori
}

\subsection{Signs and Symptoms}

Paraclinoid aneurysm is defined as an aneurysm that originates at the internal carotid artery (ICA) distal to the proximal dural ring (PDR) and proximal to the posterior communicating artery (PCoA), which means both ophthalmic and clinoidal segments of the ICA. Patients with these aneurysms present with retro-orbital or supraorbital pain and varying degrees of visual field constriction and/or visual acuity decline which is associated with compression of optic nerve. This nerve is typically pushed superiorly and medially, which manifests as a unilateral inferomedial (lower nasal) quadrantanopsia [1]. In addition, paraclinoid aneurysms may manifest as progressive diplopia due to compression of the cranial nerves involved in ocular movement caused by aneurysm growth. Unruptured paraclinoid aneurysm has a low risk of rupture compared to other types of cerebral aneurysm. The surgical indications for unruptured paraclinoid aneurysm should consider this low rupture risk as well as several other factors such as the aneurysm shape, the aneurysm size, and how old the patient.

\subsubsection{Case Illustration One}

A 56-year-old female was admitted to our service due to the progressive visual field deficit consisting of inferomedial quadrantanopsia (Fig. 13.1) with retro-orbital pain. We ordered a 3D-CT angiography, and it demonstrated a right ICA aneurysm with the dome projecting superiorly (Fig. 13.2).

\subsubsection{Case Illustration Two}

A 64-year-old female was brought to the hospital due to a severe retro-orbital pain. 3D-CT angiography demonstrated a large left ICA-ophthalmic artery aneurysm (Fig. 13.3a). The aneurysm dome projected medio-superiorly. Postoperative 3D-CT angiography showed complete clipping was achieved (Fig. 13.3b).

\footnotetext{
N. Otani $(\bowtie) \cdot$ T. Toyooka $\cdot$ K. Mori

Department of Neurosurgery, National Defense Medical College, Tokorozawa, Saitama, Japan e-mail: naotani@ndmc.ac.jp; toyo@ndmc.ac.jp; kmori@ndmc.ac.jp
} 

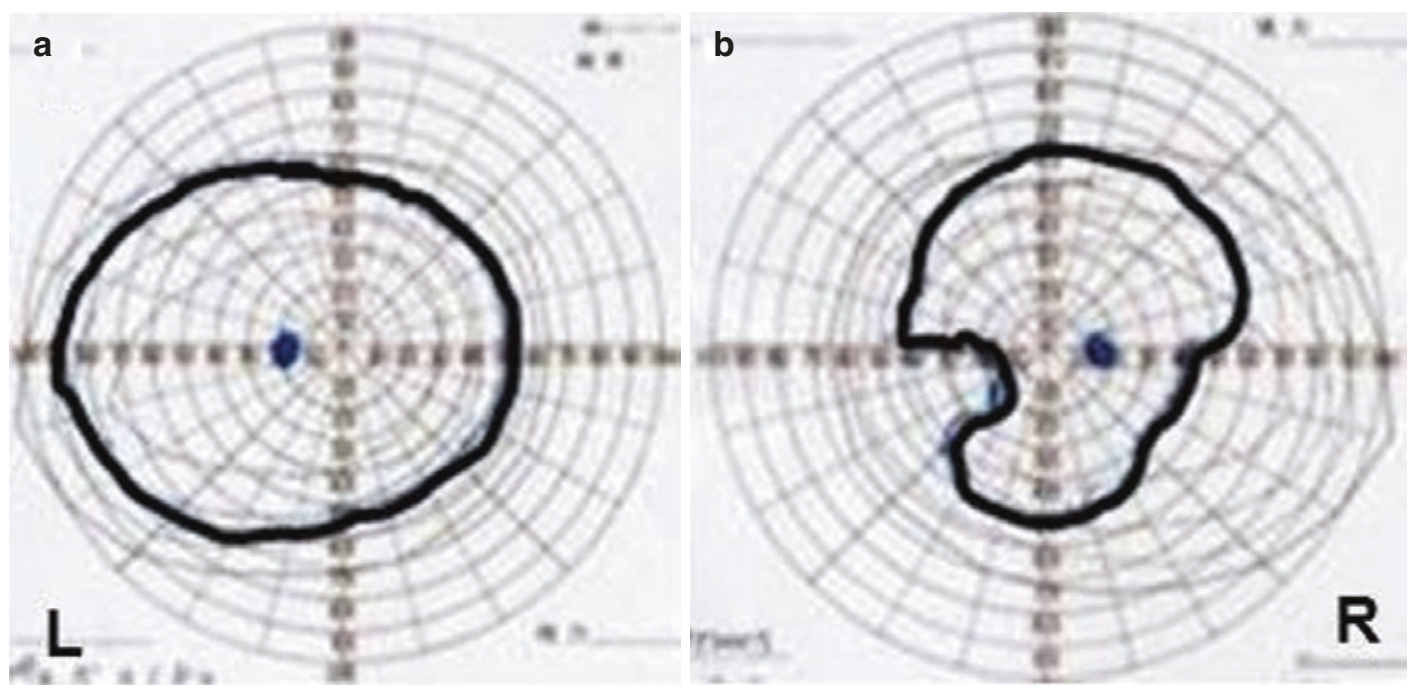

Fig. 13.1 Case 1. Visual field examination showing unilateral inferomedial quadrantanopsia on the right (b). Normal visual field on the left (a)
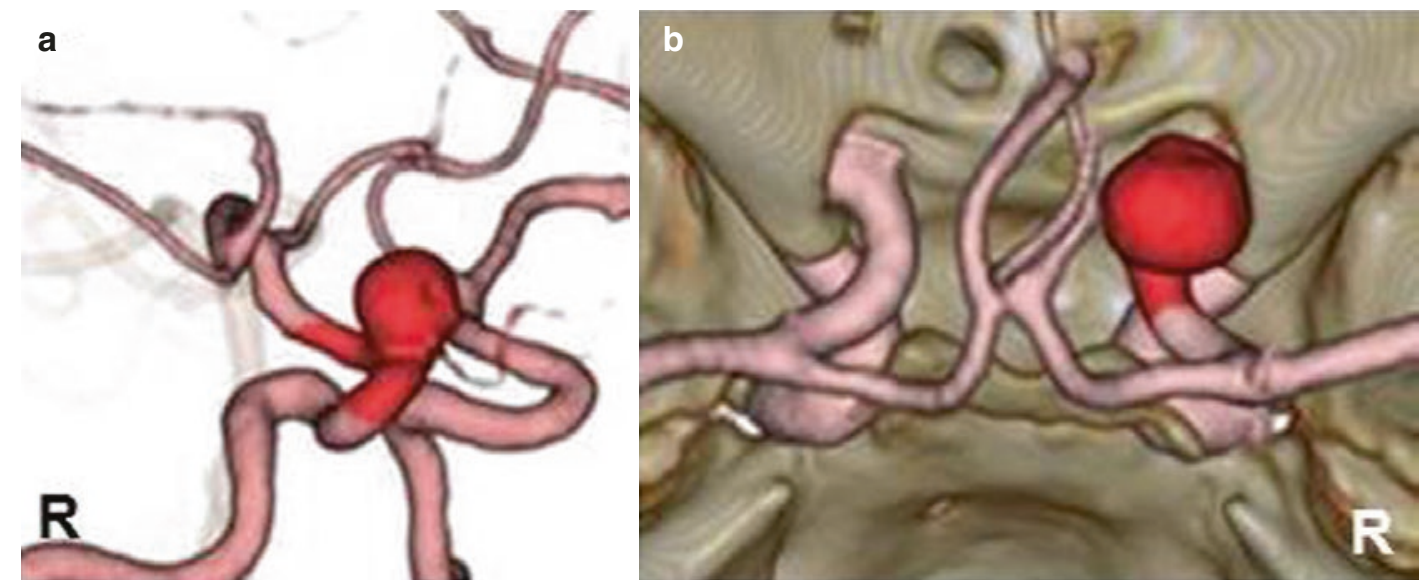

Fig. 13.2 Case 2. Three-dimensional computed tomography angiograms showing a paraclinoid aneurysm. (a) Oblique view. (b) superior view

\subsection{Investigation}

The ICA that is located along the anterior clinoid process (ACP), between proximal and distal dural ring (DDR), is called clinoid segment (usually referred as $\mathrm{C} 3$ ). After passing the distal dural ring (DDR), the ICA actually runs in the subarachnoid space. From DDR to the origin of the PCoA is called ophthalmic segment. In order to make it clear, the term paraclinoid aneurysm is the aneurysm that originates from the ICA between the PDR and the PCoA (or in the other 

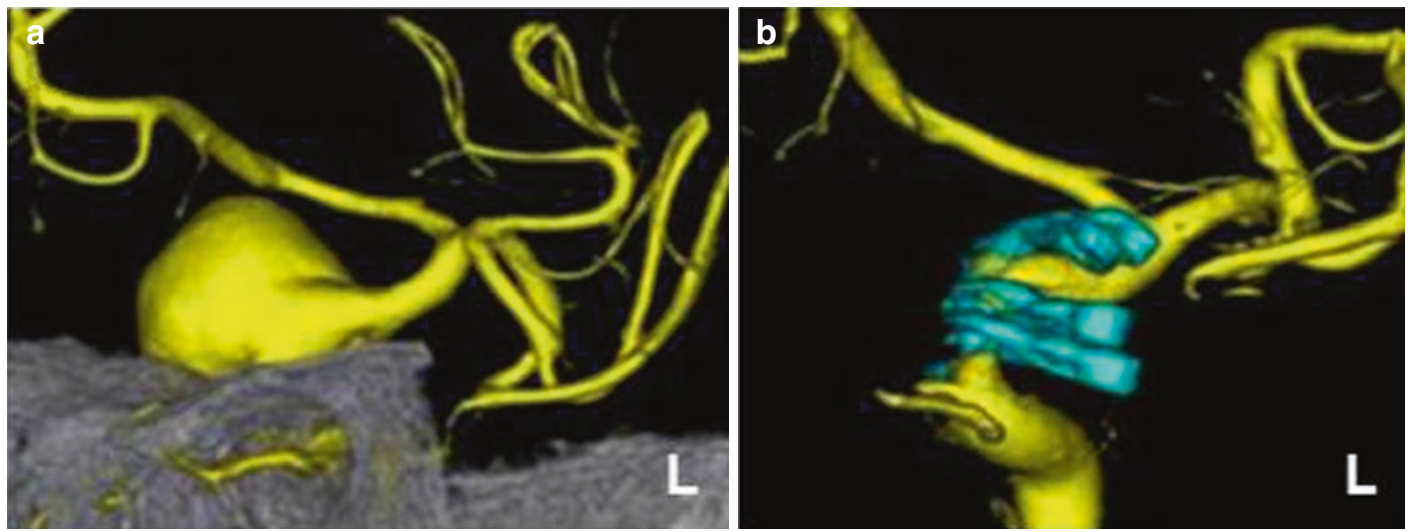

Fig. 13.3 Case 2. (a) Preoperative three-dimensional computed tomography angiogram showing a paraclinoid aneurysm. (b) Postoperative three-dimensional computed tomography angiogram showing complete clipping of the aneurysm words, between the clinoid and ophthalmic segments). Paraclinoid aneurysms can be classified as ophthalmic aneurysm (which arise from superior part of ICA), superior hypophyseal aneurysm (which arise from the inferomedial part of ICA), supra-clinoid aneurysm which arise from anterior wall of ICA, and the infraclinoid aneurysm which arise from posterior wall of ICA. These aneurysms are usually surrounded by bony and neurovascular anatomy, namely, ophthalmic artery, optic nerve, oculomotor nerve, and ACP (Figs. 13.4 and 13.5). The most important step in the dissection for this aneurysm is to release the aneurysm dome from optic nerve and ACP.

\subsection{Preoperative Preparation}

\subsubsection{Neuroimaging Examinations}

Bone CT is useful to investigate paraclinoid aneurysm. The size, shape, and pneumatization of the ACP and surrounding sinus, such as sphenoid and ethmoid, all should be assessed for safe clinoidectomy. Calcification of the proximal ICA should be examined for safe proximal control just before clip application.

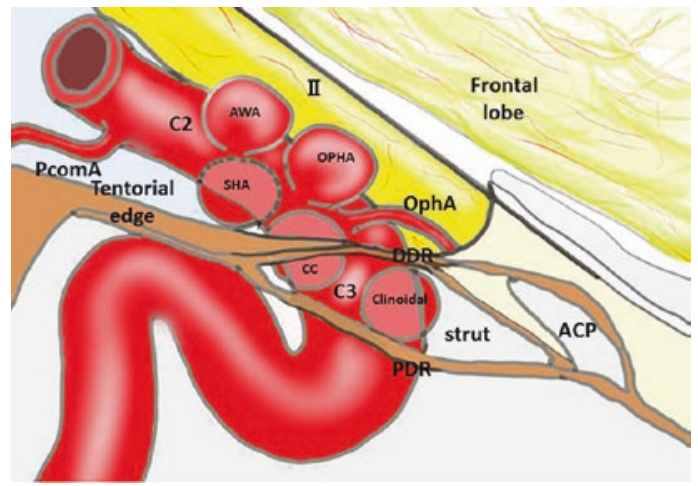

Fig. 13.4 Illustration of paraclinoid aneurysm. The clinoid segment (C3) of the internal carotid artery (ICA) runs along the anterior clinoid process (ACP), passing between the proximal (PDR) and distal dural rings (DDR), and the ophthalmic segment (C2) courses from the DDR to the posterior communicating artery (PCoA). Paraclinoid aneurysm originates from the ICA between the PDR and the PCoA (clinoid and ophthalmic segments). Paraclinoid aneurysms are classified as ophthalmic, anterior wall type located on the anterior wall of the ICA, superior hypophyseal, carotid cave type, and clinoidal segment aneurysms located on the infero-medial wall of the ICA, and variant aneurysms. Paraclinoid aneurysms are surrounded by many important osseous and neurovascular structures such as the ACP, optic nerve (II), oculomotor nerve, ICA, and ophthalmic artery (OphA). AWA anterior wall aneurysm, $O p h A$ ophthalmic artery aneurysm, $S H A$ superior hypophyseal artery aneurysm, $C C$ carotid cave aneurysm, clinoidal clinoidal segment aneurysm, strut optic strut 


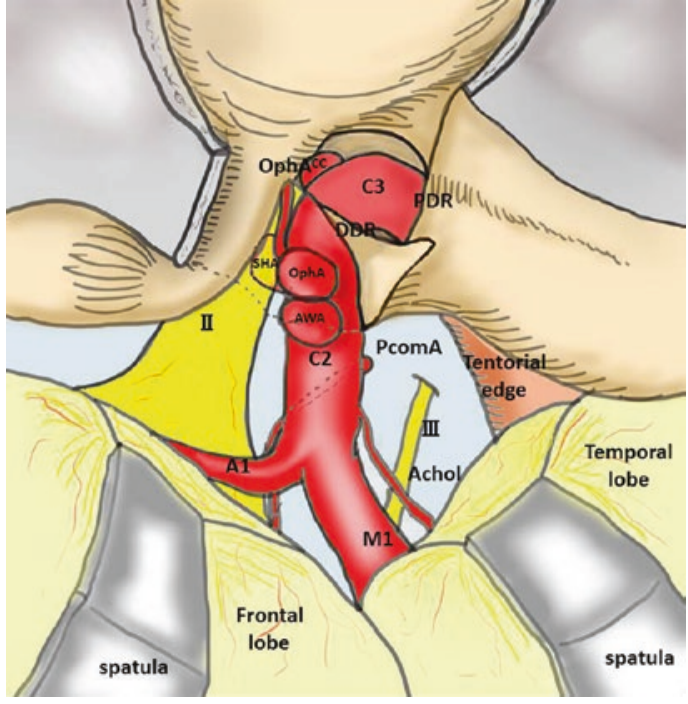

Fig. 13.5 Illustration of treatment of paraclinoid aneurysm via the anterolateral approach. Incision of the falciform ligament to the optic nerve is made to mobilize the optic nerve (II). Additional incision is made across the distal dural ring (DDR) to expose and identify the origin of the ophthalmic artery (OphA) and to mobilize the internal carotid artery (ICA). III oculomotor nerve, C2 ophthalmic segment of the ICA, $C 3$ clinoid segment of the ICA, $P C o A$ posterior communicating artery, Achol anterior choroidal artery, $A W A$ anterior wall aneurysm, $O p h A$ ophthalmic artery aneurysm, $S H A$ superior hypophyseal artery aneurysm, $C C$ carotid cave aneurysm, clinoidal clinoidal segment aneurysm, $P D R$ proximal dural ring, $A 1$ horizontal portion of the anterior cerebral artery, $M 1$ horizontal portion of the middle cerebral artery
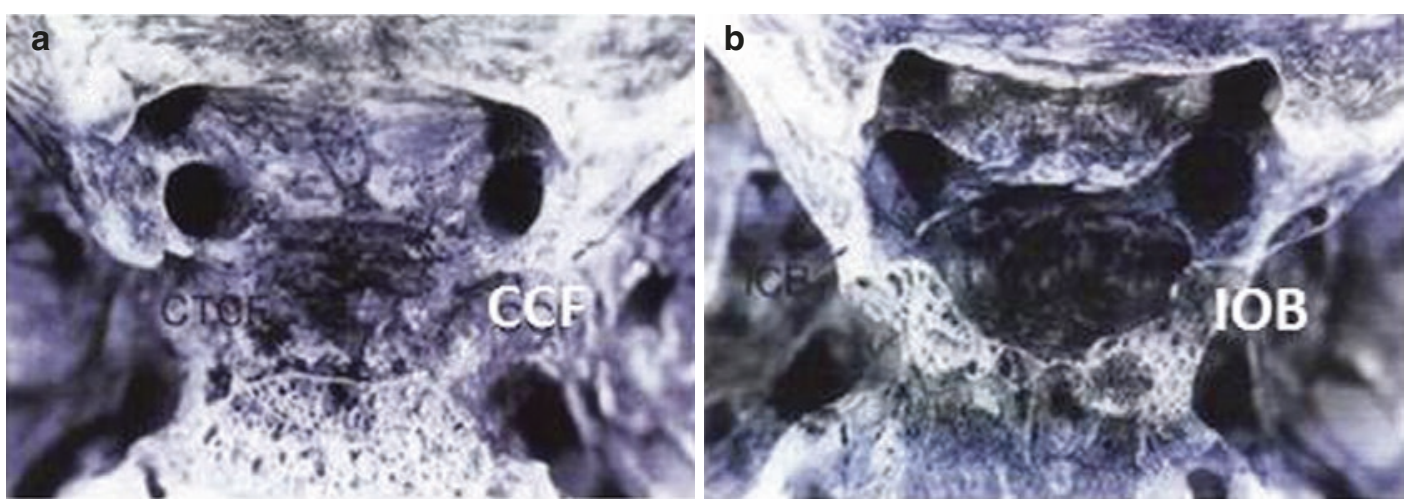

Fig. 13.6 Contraindications for anterior clinoidectomy such as caroticoclinoid foramen (a, CCF) and interclinoid osseous bridge (b, IOB) should be assessed preoperatively tomy extradurally are caroticoclinoid foramen (Fig. 13.6a) and the interclinoid ligament (Fig. 13.6b); both should be assessed preoperatively [2]. 3D-CT angiography with or without DSA are mandatory to investigate paraclinoid aneurysm. Meningo-orbital artery (MOA) forming the main collateral to the retinal artery cannot be resected. The development of the superficial Sylvian vein and the circulation pattern of the sphenoparietal sinus should be evaluated in the venous phase, which may show contraindication for dural peeling of the lateral cavernous sinus in extradural anterior clinoidectomy. The role of (MR) imaging is helpful to show the relationship of the dome and the optic nerve, using constructive interference in steadystate sequence.

\subsubsection{Suction Decompression}

Suction decompression via the cervical carotid artery and detachment of aneurysm from the nearby anatomical structures (e.g., optic nerve) are necessary for big and giant aneurysm. We also may prepare the carotid at cervical level to use it as a proximal control or angiography during surgery (Fig. 13.7).
Few contraindications to do anterior clinoidec- 

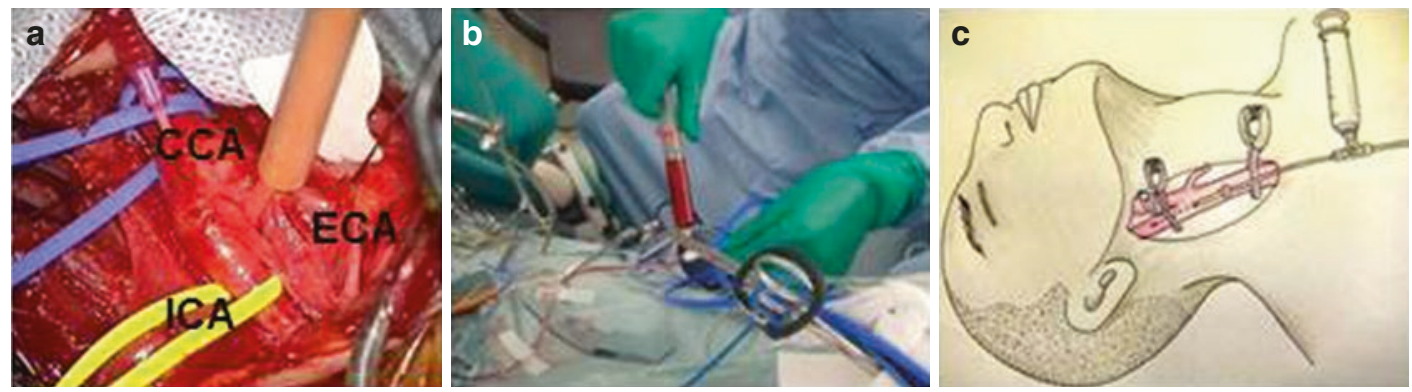

Fig. 13.7 Suction decompression (b and c) to expose and secure the cervical carotid artery (a) also helps proximal control of the internal carotid artery (ICA). CCA common carotid artery, ECA external carotid artery

\subsubsection{Bypass Procedure (Revascularization)}

High-flow bypass should be prepared, if necessary. If the aneurysm cannot be clipped for any technical reasons, either it calcified at the neck area or sclerotic, then bypass surgery is necessary from extracranial carotid artery to M2 segment of MCA; we may use the radial artery or saphenous vein.

\subsubsection{Intraoperative Angiography}

During surgery, it is necessary to confirm complete secure of the aneurysm and also needs to confirm the preservation of branches and parent artery flow, by using the intraoperative angiography. Therefore, the radiolucent Mayfield head clamp is helpful.

\subsubsection{Spinal Drainage}

There is some advantages of spinal drainage to enhance the brain relaxation during surgery, which enable to get more wide epidural space during extradural surgical procedure. In addition, these procedures may reduce the occurence of CSF leakage postoperatively.

\subsubsection{Intraoperative Monitoring}

Intraoperative monitoring of the motor-evoked potential (Fig. 13.8b) and visual-evoked potential (Fig. 13.8a) is mandatory for safe clipping.

\subsubsection{Instruments for Anterior Clinoidectomy}

A 6-mm coarse burr drill is useful for removing the lateral part of sphenoid bone. Sharp dissectors are needed for gently detaching lateral wall of cavernous sinus. A 2-mm or 3-mm coarse burr drill is useful for drilling of the ACP. In addition, an irrigation suction system, micro-rongeur, and micro-punch are necessary to minimize mechanical or avoid heating the optic nerve.

\subsubsection{Approach}

As mentioned before, removing of ACP is one of the very important steps to have a safe clipping of paraclinoid aneurysm. The pterional transSylvian approach with intradural anterior clinoidectomy and the Dolenc approach of combined extradural and intradural clinoidectomy are usually used to treat paraclinoid aneurysms, but both approaches have advantages and 

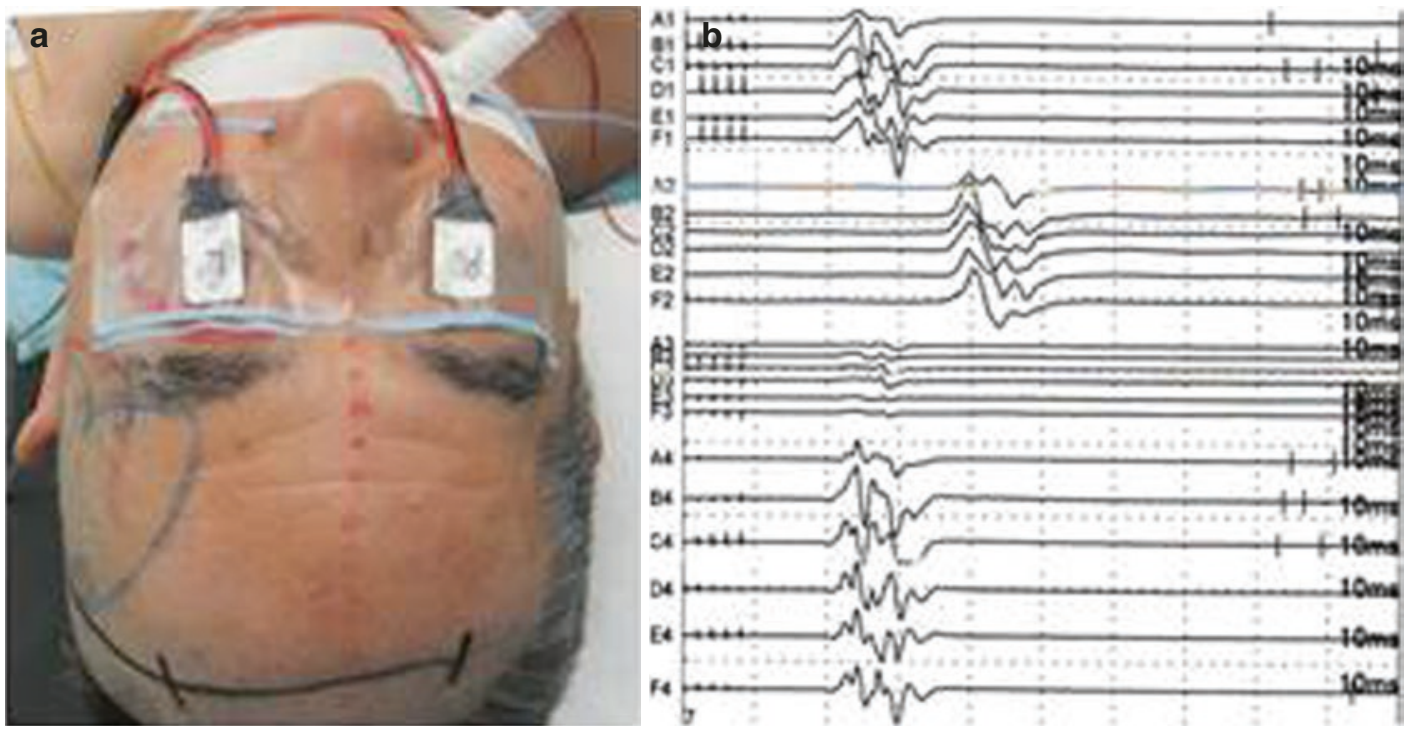

Fig. 13.8 Preparation for intraoperative visual-evoked potential (a) and motor-evoked potential monitoring (b)

disadvantages [3]. The pterional trans-Sylvian approach with intradural anterior clinoidectomy has the advantage of adequate exposure to dissect the aneurysm but carries a critical risk of destructive injury of the ICA, optic nerve, and aneurysm, even if protected by the dural flap. The extradural temporopolar approach is an epidural approach involving the moving of temporal lobe posteriorly with the dura mater $[4,5]$ and includes extradural anterior clinoidectomy, optic canal opening, detaching lateral wall of cavernous sinus, and then retraction of temporal lobe over the dura mater. Such epidural temporal lobe retraction is less likely to cause temporal lobe contusion and critical injury during anterior clinoidectomy.

\subsection{Steps of the Surgery}

\subsubsection{Patient Position}

After the patient was put to sleep and placement of the lumbal drain for CSF aspiration, the patient is placed in supine position and head rotated to contralateral side of operative field about $30^{\circ}$, slightly extended to have the anterior cranial fossa perpendicular to the floor. Neck should be

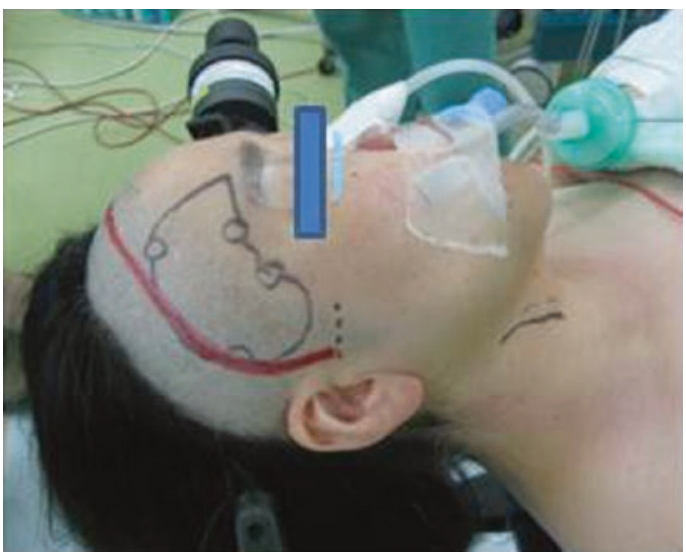

Fig. 13.9 Scheduled skin incision and frontotemporal craniotomy

slightly extended and elevated to avoid venous congestion. Additional slight lateral extension of the head helps to expose the carotid ICA (Fig. 13.9).

\subsubsection{Skin Incision}

The skin is cut half coronal and then inter-fascial separation is performed. Retract the temporal muscle to inferior without incision. The cervical 
common carotid artery, ICA and ECA will be prepared for many purposes later on such as proximal control, angiography during surgery, suction decompression if necessary, or even highflow bypass if the patient needs it.

\subsubsection{Craniotomy}

Frontotemporal (pterional) bone flap is opened until supraorbital notch, the squama part of the temporal bone is removed until we expose the middle cranial floor, and on top of that we could add osteotomy of orbitozygomatic, if orbitozygomatic approach is needed for a large or giant aneurysm. It is easier to cut the orbitozygomatic bar in the two-piece method. Subperiosteal dissection is done for frontal and temporal. Then drill the lesser wing of the sphenoid until it becomes flat, and combine with the use of a rongeur; it is necessary to expose the meningoorbital band. Keep dissecting the dura, and expose the superior orbital fissure (SOF) and foramen rotundum (Fig. 13.10). After the roof of the SOF is exposed, we need to identify intersection of periosteal dura and dura propria of temporal lobe. The bone around the meningo-orbital band needs to be drilled, and then incise the band about 4-mm length (Fig. 13.11). Gently detach the dura propria from the lateral wall of the SOF in order to expose ACP from extradural (Fig. 13.12). It is important to preserve sphenoparietal sinus while detaching dura propria and

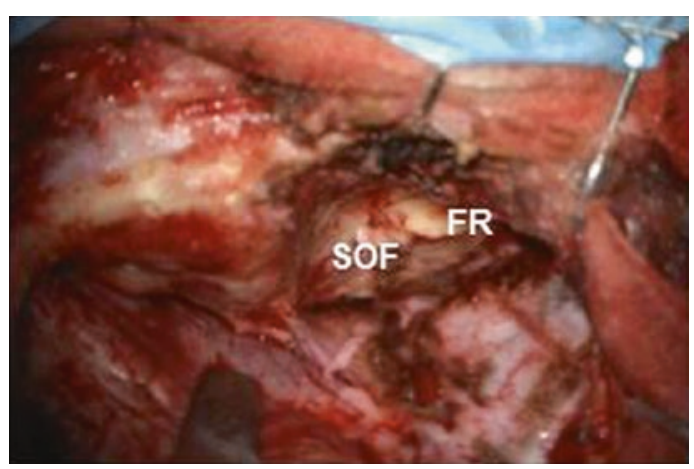

Fig. 13.10 After frontotemporal craniotomy. The foramen rotundum (FR) and superior orbital fissure (SOF) are exposed

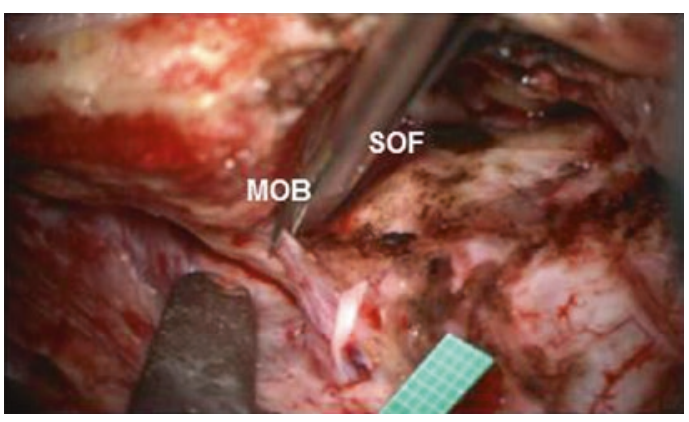

Fig. 13.11 Cutting the meningo-orbital band (MOB). SOF superior orbital fissure

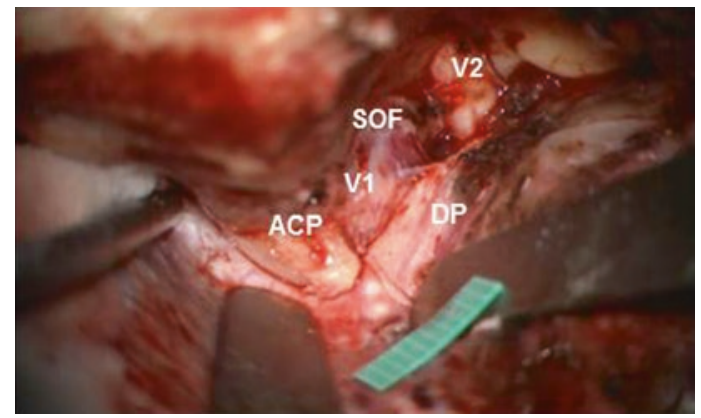

Fig. 13.12 After peeling of the dura propria (DP) of the temporal dura mater and epidural exposure of the anterior clinoid process (ACP). V1 ophthalmic branch of the trigeminal nerve, $V 2$ maxillary branch of the trigeminal nerve, $S O F$ superior orbital fissure

stop detaching at location where the cavernous sinus receives the blood from the sphenoparietal sinus. It is done to avoid venous congestion postoperatively.

\subsubsection{Opening the Optic Canal and then Complete Removal of ACP}

Orbital roof is drilled gently until it is as thin as the eggshell layer. Following the SOF laterally, identify the base of ACP, and then open the orbit at its posterior part. Expose the periorbital, and detach it from inside of orbit with preservation of the periorbital fat. The optic nerve is identified as it exits the optic canal. Start drilling the ACP from lateral to medial part; having plenty of saline irrigation is needed to avoid heat injury. Open the optic canal at medial part of ACP by using 


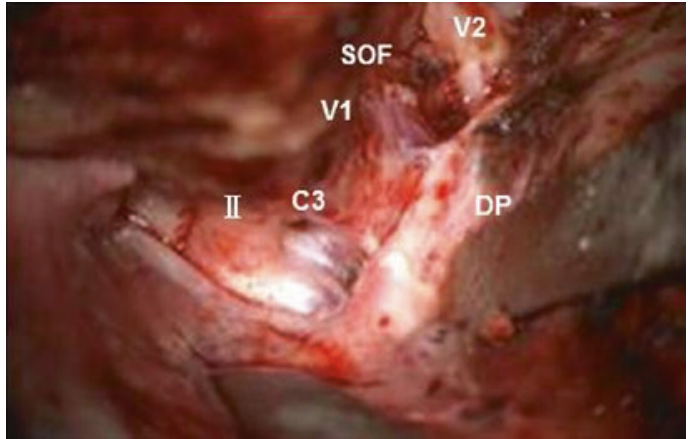

Fig. 13.13 Completion of epidural procedures after removal of the anterior clinoid process and opening of the optic canal. II optic nerve, VI ophthalmic branch of the trigeminal nerve, $V 2$ maxillary branch of the trigeminal nerve, $C 3$ clinoid segment of the internal carotid artery, $D P$ dura propria, $S O F$ superior orbital fissure

micro-punch, with thinning of the bone along the medial and anterior surfaces of the clinoid process. Drill the core of ACP by using 2-mm coarse diamond, and then it's dissected away from carotidoculomotor membrane. At the end, we may gently remove $\mathrm{ACP}$, and it usually will be followed with cavernous sinus bleeding and easily be managed with cottonoid and fibrin glue (Fig. 13.13).

\subsubsection{Removing Optic Strut to Insert the Clip Blades}

The half opening of optic canal can be enlarged by a micro-punch, and the rest of the optic strut (between the opened clinoid space and optic canal) can be taken by small diamond drill or using a fine micro-punch to get a space for clip blade insertion.

\subsubsection{Open the Dura and Sylvian Fissure Dissection}

The dura is opened following the Sylvian fissure and then extended inferiorly and medially to reach the optic nerve level. Wide dissection of Sylvian fissure helps to minimize brain retraction when we need to expose the optic nerve and ICA. During the dissection, it is necessary to identify the PCoA and anterior choroidal artery.
In addition, horizontal portion of anterior cerebral artery must be dissected.

\subsubsection{Cutting Falciform Ligament and DDR}

Sharp cut of falciform ligament to the optic nerve is made with microscissors in order to be able to move the optic nerve (Fig. 13.14). Another cut is made across the DDR; the purpose is to find ophthalmic artery origin and to get more room to mobilize the ICA (Fig. 13.15).

\subsubsection{Clip Application}

The dome projection and the relationships between the parent artery and aneurysm dome

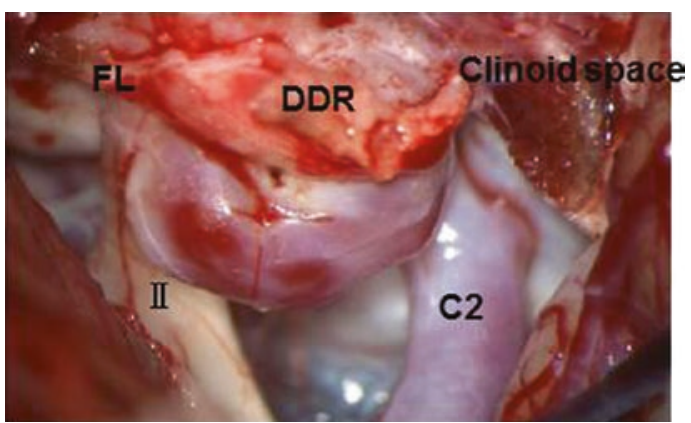

Fig. 13.14 The falciform ligament (FL) and the distal dural ring (DDR) are incised. $I I$ optic nerve, $C 2$ ophthalmic segment of the internal carotid artery

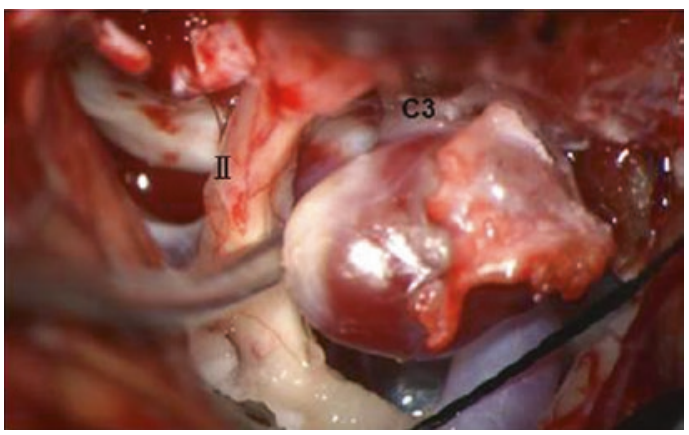

Fig. 13.15 Additional incision is made across the distal dural ring to expose and identify the origin of the ophthalmic artery and to mobilize the internal carotid artery (ICA). II optic nerve, $C 3$ clinoid segment of the ICA 


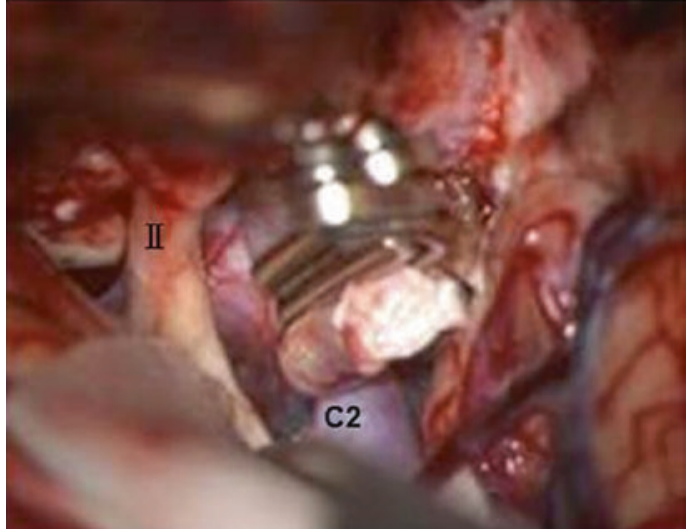

Fig. 13.16 After neck clipping of the paraclinoid aneurysm. II optic nerve, $C 2$ ophthalmic segment of the internal carotid artery

depend on the clip selection [1, 6, 7]. In most situation, the supra-clinoid-type or small ophthalmic artery aneurysms just require a simple straight or slightly curve clip (Fig. 13.16). Sidecurved clips also can be used for its configuration. In contrast, angled fenestrated clips are typically required for infra-clinoid-type aneurysms such as superior hypophyseal artery aneurysms or carotid cave aneurysms, particularly large or giant aneurysms.

\subsubsection{Suction Decompression (If Necessary)}

Suction decompression is useful for detachment of aneurysm dome from the adjacent neurovascular structure such as optic nerve (Fig. 13.17).

\subsubsection{Hemostasis and Closure}

If during the clinoid drilling we get in to the ethmoidal cells, the chance of CSF leakage is high. It is necessary to pack the ethmoidal cells with periosteum or fat and then sealed with fibrin glue. Skull is fixed with titanium plates, a subcutaneous drain is placed, and the wound is closed as usual.

\subsection{Expert Opinion/Suggestion to Avoid Complication}

\subsubsection{Avoiding Visual Disturbance (Optic Nerve Injury)}

Continuous irrigation with cold water is mandatory during drilling procedures to avoid heat injury. While dissecting the aneurysm from optic nerve, meticulous manipulation and minimal use of bipolar coagulation are extremely important to spare the microvessels of the optic nerve. Suction decompression is also useful to perform complete dissection safely without optic nerve injury.

\subsubsection{Avoiding CSF Leakage (Rhinorrhea)}

The presence of sphenoidal sinus extension into the ACP (pneumatization) should be assessed preoperatively. During drilling of the ACP, avoid going in to optic recess of sphenoid sinus or even ethmoid air cells. The dural ring needs to be sealed with temporal fascia and fibrin glue. Spinal drainage is useful to prevent CSF leakage postoperatively, and it should be maintained for several day.

\subsubsection{Intraoperative Rupture of the Aneurysm}

The cervical ICA is secured for proximal control.

\subsubsection{Occlusion and Injury of Ophthalmic Artery or ICA}

The course of ophthalmic artery must be confirmed just before cutting the DDR and after clip placement. Intraoperative angiography or indocyanine green videoangiography is useful to check preservation of the parent artery and complete clipping. 

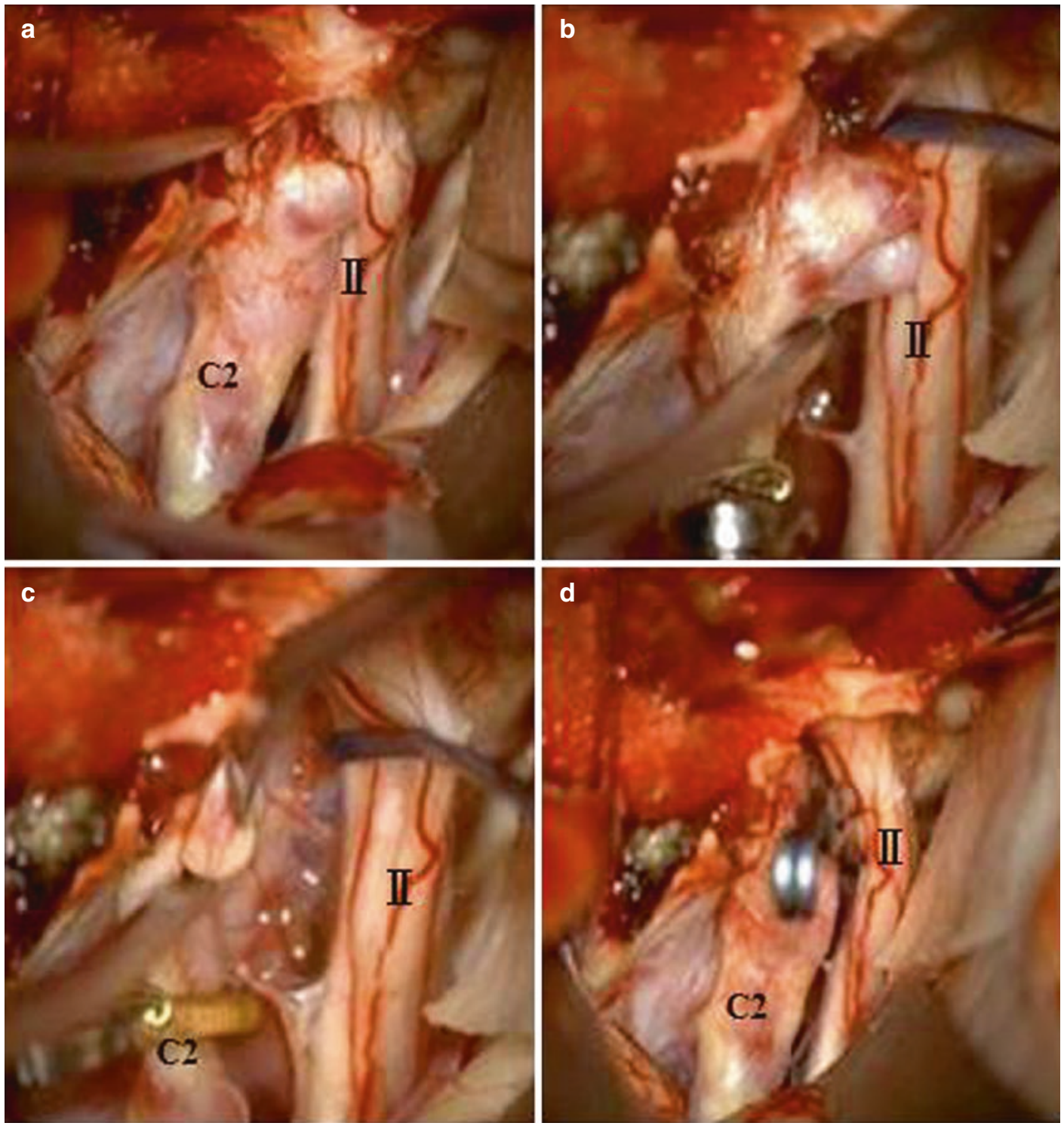

Fig. 13.17 Suction decompression (b and $\mathbf{c}$ ) is useful for detachment of the aneurysm dome from the surrounding

anatomical structures such as the optic nerve (II) (a) and for complete clipping (d). C2 ophthalmic segment of the internal carotid artery

\subsection{Things to Be Observed and Postoperative Care/ Follow-Up}

3D-CT angiography or MR angiography is for postoperative confirmation of complete aneurysm occlusion and ensures patency of parent artery. Close observation and treatment are needed if vasospasm occurs in patients with

ruptured aneurysm. CSF leakage and visual disturbance should be closely monitored postoperatively.

\section{References}

1. Day AL. Aneurysms of the ophthalmic segment. A clinical and anatomical analysis. J Neurosurg. 1990;72:677-91. 
2. Erturk M, Kayalioglu G, Gousa F. Anatomy of the clinoidal region with special emphasis on the caroticoclinoid foramen and interclinoid osseous bridge in a recent Turkish population. Neurosurg Rev. 2004;27:22-6.

3. Dolenc VV. A combined epi- and subdural direct approach to carotid-ophthalmic artery aneurysms. J Neurosurg. 1985;62:667-72.

4. Day JD, Giannotta SL, Fukushima T. Extradural temporopolar approach to lesions of the upper basilar artery and infrachiasmatic region. J Neurosurg. 1994;81:230-5.
5. Mori K, Yamamoto T, Nakao Y, Esaki T. Surgical simulation of extradural anterior clinoidectomy through the trans-superior orbital fissure approach using a dissectable three-dimensional skull base model with artificial cavernous sinus. Skull Base. 2010;20:229-36.

6. Giannota SL. Ophthalmic segment aneurysm surgery. Neurosurgery. 2002;50:558-62.

7. Heros RC, Nelson PB, Ojemann RG, Crowell RM, DeBrun G. Large and giant paraclinoid aneurysms: surgical techniques, complications and results. Neurosurgery. 1983;12:153-63.

Open Access This chapter is licensed under the terms of the Creative Commons Attribution 4.0 International License (http://creativecommons.org/licenses/by/4.0/), which permits use, sharing, adaptation, distribution and reproduction in any medium or format, as long as you give appropriate credit to the original author(s) and the source, provide a link to the Creative Commons license and indicate if changes were made.

The images or other third party material in this chapter are included in the chapter's Creative Commons license, unless indicated otherwise in a credit line to the material. If material is not included in the chapter's Creative Commons license and your intended use is not permitted by statutory regulation or exceeds the permitted use, you will need to obtain permission directly from the copyright holder. 